\title{
TU/e EN⿴HONE

\section{Binary colloidal nanoparticle concentration gradients in a centrifugal field at high concentration}

\section{Citation for published version (APA):}

Xu, X., Franke, T., Schilling, K., Sommerdijk, N. A. J. M., \& Cölfen, H. (2019). Binary colloidal nanoparticle concentration gradients in a centrifugal field at high concentration. Nano Letters, 19(2), 1136-1142. https://doi.org/10.1021/acs.nanolett.8b04496

DOI:

10.1021/acs.nanolett.8b04496

Document status and date:

Published: 13/02/2019

\section{Document Version:}

Accepted manuscript including changes made at the peer-review stage

\section{Please check the document version of this publication:}

- A submitted manuscript is the version of the article upon submission and before peer-review. There can be important differences between the submitted version and the official published version of record. People interested in the research are advised to contact the author for the final version of the publication, or visit the $\mathrm{DOI}$ to the publisher's website.

- The final author version and the galley proof are versions of the publication after peer review.

- The final published version features the final layout of the paper including the volume, issue and page numbers.

Link to publication

\section{General rights}

Copyright and moral rights for the publications made accessible in the public portal are retained by the authors and/or other copyright owners and it is a condition of accessing publications that users recognise and abide by the legal requirements associated with these rights.

- Users may download and print one copy of any publication from the public portal for the purpose of private study or research.

- You may not further distribute the material or use it for any profit-making activity or commercial gain

- You may freely distribute the URL identifying the publication in the public portal.

If the publication is distributed under the terms of Article 25fa of the Dutch Copyright Act, indicated by the "Taverne" license above, please follow below link for the End User Agreement:

www.tue.nl/taverne

Take down policy

If you believe that this document breaches copyright please contact us at:

openaccess@tue.nl

providing details and we will investigate your claim. 


\title{
Binary colloidal nanoparticle concentration
}

\section{gradients in a centrifugal field at high concentration}

\author{
X. Xu ${ }^{123}$, T. Franke ${ }^{2}$, K. Schilling ${ }^{2}$, N.A.J.M. Sommerdijk ${ }^{1}$, H. Cölfen ${ }^{3 *}$ \\ ${ }^{1}$ Laboratory of Materials and Interface Chemistry \& Centre for Multiscale Electron Microscopy, \\ Eindhoven University of Technology, 5600 MB Eindhoven, Netherlands \\ ${ }^{2}$ Nanolytics GmbH, Am Mühlenberg 11, 14476 Potsdam, Germany \\ ${ }^{3}$ Physical Chemistry, University of Konstanz, Universitätsstr 10, Box 714, 78457 Konstanz, Germany
}

ABSTRACT: Binary colloidal nanoparticles have been found to form different types of crystalline phases at varied radial positions in a centrifugal field by Chen et al (ACS nano 2015, 9, 6944-50.). The variety of binary phase behaviors resulted from the two different nanoparticle concentration gradients but to date the gradients can only be empirically controlled. For the first time, we are able to measure, fit and simulate binary hard sphere colloidal nanoparticle concentration gradients at high particle concentration up to 30 vol\%, which enables tailor-made gradients in a centrifugal field. By this means, a continuous range of binary particle concentration ratios can be accessed in one single experiment to obtain an extended phase diagram. By dispersing two differently sized silica nanoparticles labeled with two different fluorescence dyes in a refractive index matching solvent, we can use a Multi-Wavelength Analytical Ultracentrifuge (MWL-AUC) to measure the individual concentration gradient for each particle size in sedimentation-diffusion equilibrium. The influence of the remaining slight turbidity at high 
concentration can be corrected using the MWL spectra from the AUC data. We also show that the experimental concentration gradients can be fitted using a non-interacting non-ideal sedimentation model. By using these fitted parameters, we are able to simulate nanoparticle concentration gradients, which agreed with the subsequent experiments at a high concentration of 10 vol\% and thus allowed for the simulation of binary concentration gradients of hard sphere nanoparticles in preparative ultracentrifuges (PUC). Finally we demonstrated that by simulating the concentration gradients in PUC, a continuous and extended binary nanoparticle phase diagram can be obtained by simply studying the structure evolution along the centrifugal field for one single sample instead of a large number of experiments with discrete compositions in conventional studies.

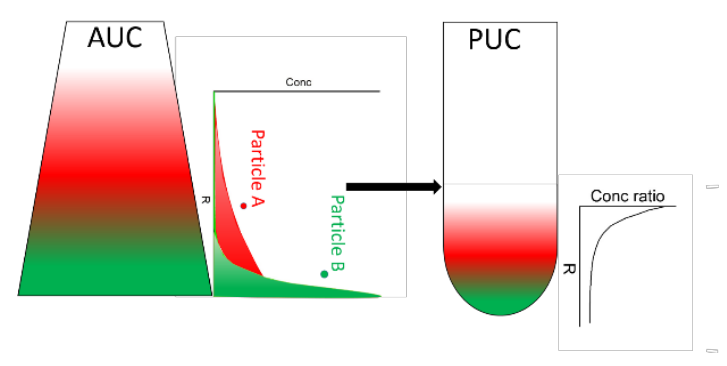

KEYWORDS: Binary nanoparticle concentration gradients, high particle concentration, sedimentationdiffusion equilibrium, binary nanoparticle phase diagram.

Colloidal nanoparticles are promising building blocks for constructing a wide variety of advanced materials, such as biological sensors ${ }^{1,2}$, catalysts ${ }^{3}$ and fuel cells $s^{4,5}$. Of particular interest are colloidal crystals $^{6,7}$ which can be used as photonic materials ${ }^{8,9}$ for modulating light flows. In the recent years, an increasing interest has been focused on binary colloidal crystals ${ }^{10,11}$, where a large variety of complicated crystalline structures has been experimentally observed ${ }^{12,13}$ and computationally 
predicted $^{14,15}$. These fascinating crystalline structures provide an excellent possibility for studying phase diagrams and testing many body statistical physics ${ }^{16-18}$.

The construction of binary colloidal crystals is currently a challenge since the crystallization process is controlled by a delicate balance of several nanoparticle properties, such as particle softness, concentration ratio, size ratio and charge ratio ${ }^{10-12}$. Among these parameters, the concentration ratio is easiest to tune but it requires a large amount of experiments with discrete compositions to observe all the possible phases including different crystalline and amorphous structures. For instance, in the recent years, the confinement was widely employed to induce the binary nanoparticle superlattice formation ${ }^{11,}$ 19, 20. Although the superlattice structure was well-ordered over a large range, only one type of ordering was present in each experiment due to the fixed concentration ratio. Recently, a large variety of binary crystalline phases, including kinetic structures, have been simultaneously observed in a single experiment in a centrifugal field by Chen et $\mathrm{al}^{12}$. Different binary nanoparticle phases were formed at various radial positions due to different radial concentration gradients of two differently sized particles in a centrifugal field, while the unusual kinetic crystalline phases might be formed due to kinetic spatial confinement and instability thresholds associated with the concentration gradients ${ }^{21}$ or the highly charged surface ${ }^{22}$. However, the radial concentration gradients were not yet experimentally accessible in an Analytical Ultracentrifugation (AUC) experiment because 1) the high refractive index difference between nanoparticles and water causes strong turbidity and 2) the two differently sized nanoparticles cannot be distinguished by optical detectors. Therefore, the desired correlation between the concentration gradients of two differently sized nanoparticles and the different binary colloidal phase behaviors could not be established. Besides, a very important open question is if these concentration gradients can be described by the established theory of ultracentrifugation ${ }^{23,24}$ and therefore be simulated in advance to reach the goal of tailor-made binary concentration gradients for the access to an extended and continuous phase diagram of the mixture of colloids ${ }^{25,26}$. In this letter, we report that 
the concentration gradients of binary fluorescence labeled silica nanoparticles in a refractive index matching solvent can be measured at high particle concentration up to 30 vol\% by a multi-wavelength UV-vis detector in an analytical ultracentrifuge (MWL-AUC) ${ }^{27}$. Moreover, the experimentally measured concentration gradients can be fitted to the Lamm equation ${ }^{28}$ using for example the SEDFIT ${ }^{29}$ software with a non-interacting non-ideal sedimentation model, which provides the required parameters including the sedimentation coefficient $s$, the diffusion coefficient $D$ as well as the non-ideality coefficients $k_{s}$ and $k_{D}$ for the subsequent simulation and prediction of binary nanoparticle concentration gradients. By using the simulated concentration gradients, the concentration ratio variation along the centrifugal field can be precisely determined and correlated with the binary nanoparticle phase behavior at each radial position as determined by the Scanning Electron Microscope (SEM). In this way a continuous and extended phase diagram can be obtained for a mixture of colloids from a single PUC experiment instead of performing a series of experiments with many discrete compositions ${ }^{26}$.

In order to measure binary nanoparticle concentration gradients by MWL-AUC, fluorescence labeled polyethylene glycol (PEG) stabilized silica nanoparticles of two different sizes, namely $30 \mathrm{~nm}$ and $40 \mathrm{~nm}$ were synthesized according to a modified protocol from combined previous works $\mathrm{s}^{30-35}$ (The detailed synthesis process is described in SI1). Briefly, the protocol included three steps: 1 . Two dyes, namely fluorescein isothiocyanate (FITC) and rhodamine isothiocyanate (RITC), were covalently bonded to cross linking 3-aminopropyltrietho-xysilane (APTES) to form two different fluorescent cores; 2. Hydrolyzed tetraethylorthosilicate (TEOS) crosslinked and formed a shell surrounding the fluorescent cores. The smallest size of fluorescence labeled silica nanoparticle seeds was reported to reach $4.4 \mathrm{~nm}$ which was marginally larger than the free dye and different amounts of TEOS were added to tune the final nanoparticle sizes ${ }^{36,37} ; 3$. Appropriate amounts of PEG-silane polymers were covalently linked to the surface of the above-synthesized silica nanoparticles (SNPs) as steric stabilizers, not only to suppress the irreversible agglomeration during centrifugation induced by up-concentration but also to neutralize 
most surface charges so that the nanoparticles can be treated as hard spheres ${ }^{38}$. By this approach, 30 nm RITC-SNPs and 40 nm FITC-SNPs were synthesized. Moreover, a key prerequisite for a proper AUC measurement is that the turbidity of nanoparticle dispersions has to be minimized. Otherwise most incident light will be scattered and no concentration gradients can be detected by the optical system of the AUC. This issue can be solved by using a refractive-index $(n)$ matching solvent and correcting the remaining slight turbidity at high concentration using the MWL spectra in the AUC data. In this paper, a 80 vol\% glycerol and 20 vol\% water mixture was used to match the refractive index of the silica nanoparticles $(n \approx 1.45$ ) (Figure S1). It was also proven that the glycerol water mixture formed a negligible density gradient in the AUC at the applied angular velocity (The detailed calculation procedures are described in SI2).

AUC has been in long-term use as a precise method $^{39,40}$ for characterizing sedimentation-diffusion processes of colloidal nanoparticles in situ. The concentration profiles can be detected in situ by embedded interference optics or UV-vis absorbance optics ${ }^{41}$, 42 . The recent launch of multiwavelength (MWL) optical detectors ${ }^{27,43,44}$ has been allowing for the simultaneous measurement of binary or even multiple nanoparticles, given that the particles are labeled with different chromophores. In a sedimentation-diffusion equilibrium (AUC-SE) experiment, the final equilibrium is formed due to the counterbalanced sedimentation and diffusion processes in a centrifugal field ${ }^{24}$. In order to fit the experimentally obtained equilibrium concentration gradients, the SEDFIT software package ${ }^{45}$ was used. The fitting is based on is the balance of a diffusion flux $j_{\text {diff }}$ and a sedimentation flux $j_{\text {sed }}$ in a steady state given by $j_{\text {sed }}+j_{\text {diff }}=0$. Based on this principle, the barometric Boltzmann exponential ${ }^{23}$ can be derived. For fitting and simulating an experimental nanoparticle concentration gradient, a noninteracting non-ideal sedimentation model embedded in the software was used because 1) The synthesized SNPs were sterically stabilized by PEG chains of a short length ( $M w=1160 \mathrm{Da}$, circa $1 \mathrm{~nm})$ and the surface charge was mostly neutralized by the PEG-silane linkage (Zeta potential $=-6 \mathrm{mV}$ in 
water). The Van der Waals attraction was also minimized by the refractive index matching. Therefore the nanoparticles can be treated as hard spheres ${ }^{38} ; 2$ ) The non-ideality effect must be taken into account at high concentration ${ }^{46,47}$ by including the non-ideality coefficients $k_{s}$ and $k_{\mathrm{D}}$. Both thermodynamic and hydrodynamic non-ideality ${ }^{41}$ were taken into account by: $s=s_{0} /\left(1+k_{s} * c\right)$ and $D=D_{0} /\left(1+k_{d} * c\right)$ where $s_{0}$ and $D_{0}$ are the sedimentation and diffusion coefficients at infinite dilution, and $s$ and $D$ are the sedimentation and diffusion coefficients at concentration $c$. In the applied model, the experimental concentration profiles can be fitted, obtaining the sedimentation coefficient $s$, the diffusion coefficient $D$ as well as the non-ideality coefficients $k_{\mathrm{s}}$ and $k_{\mathrm{D}}$. The fitted parameters can be further used for subsequent simulations of binary concentration gradients with the goal to finally be able to simulate concentration gradients in a preparative ultracentrifuge where particle concentrations are not monitored anymore.

As a first step, the separate concentration gradients of $30 \mathrm{~nm}$ RITC-SNPs and $40 \mathrm{~nm}$ FITC-SNPs were formed in AUC-SE experiments (the detailed experimental set-up is described in SI3). By fitting the experimental data, $s, D, k_{\mathrm{s}}$ and $k_{\mathrm{D}}$ were obtained. More specifically, the values of $s$ and $D$ were first determined by fitting the sedimentation velocity (AUC-SV) experiments (Figure S4). Then $k_{\mathrm{S}}$ and $k_{\mathrm{D}}$ were determined by fitting the subsequent AUC-SE experiments (the detailed fitting process is described in S14). The final fitting results are shown in Figure 1. The fitting profiles agree with the experiments with randomly distributed residual signals. Quantitatively speaking, the root-mean-square deviation (RMSD) values of the concentration residuals reached only 0.037 vol\% and 0.039 vol\% and the maximum deviations were smaller than 0.05 vol\% and 0.07 vol\% for $30 \mathrm{~nm}$ and $40 \mathrm{~nm}$ SNPs separately. The $s$ and $D$ values used for the following simulations were also verified by calculating the densities $(\rho)$ and molecular masses $(M)$ of $30 \mathrm{~nm}$ and $40 \mathrm{~nm} \mathrm{SNPs}^{48}$. The calculation results agreed with the theoretical values within an acceptable relative deviation (the detailed calculation procedures and results are described in SI5). 
A

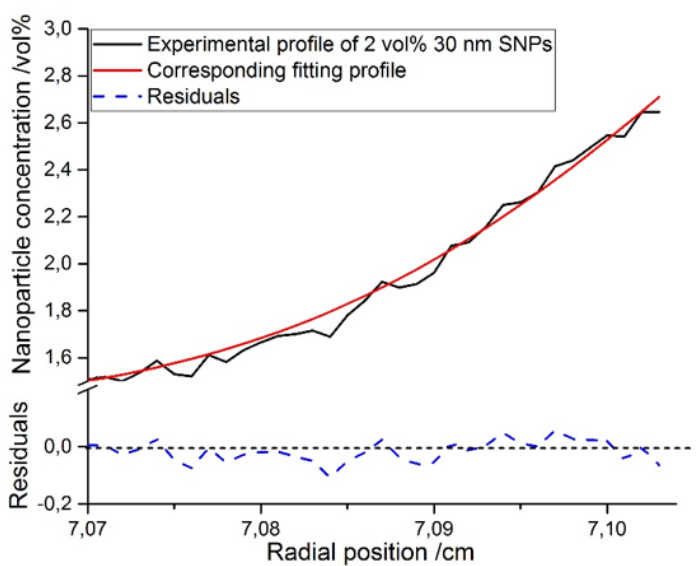

B

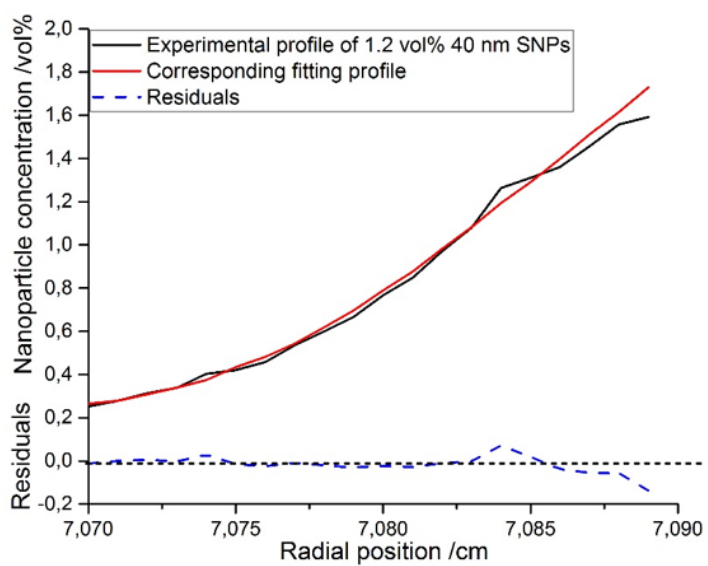

Figure 1: Experimental (black solid lines) and fitting (red solid lines) gradients for $30 \mathrm{~nm}$ RITC-SNPs of 2 vol\% (A) and $40 \mathrm{~nm}$ FITC-SNPs of 1.2 vol\% (B) in an AUC at $2800 \mathrm{rpm}, 25^{\circ} \mathrm{C}$; The blue dashed lines are the residuals which result from fitting to the experimental data. For $(A)$ and $(B)$ RMSD $=0.037$ vol\% and 0.039 vol\% respectively.

In the following, these validated fitting parameters at moderate concentrations were used for simulations of potential concentration gradients beforehand. In order to access the reliability of the simulations, two experiments were conducted for $30 \mathrm{~nm}$ and $40 \mathrm{~nm}$ SNPs separately. Firstly, the simulation reliability was proved for an extremely high concentration (30 vol\%) of $30 \mathrm{~nm}$ RITC-SNPs. In order to measure this extremely high concentration in the experiment, the optical pathlength had to be dramatically reduced to $\sim 0.1 \mathrm{~mm}$ as reported by Page et al ${ }^{49}$. As shown in Figure $2 \mathrm{~A}$, the simulation profile agrees with the experimental result, even at this extremely high concentration. Secondly, the simulation reliability was tested for a larger column height of $40 \mathrm{~nm}$ FITC-SNPs. Similarly, in Figure 2B, the simulation for a larger volume is given showing that the simulation successfully predicted the experimental concentration gradient. Overall, these experiments on the two nanoparticles demonstrated that the simulations were able to predict the concentration gradients within an acceptable deviation (RMSD < 0.04 ) regardless of nanoparticle concentration and volume. 

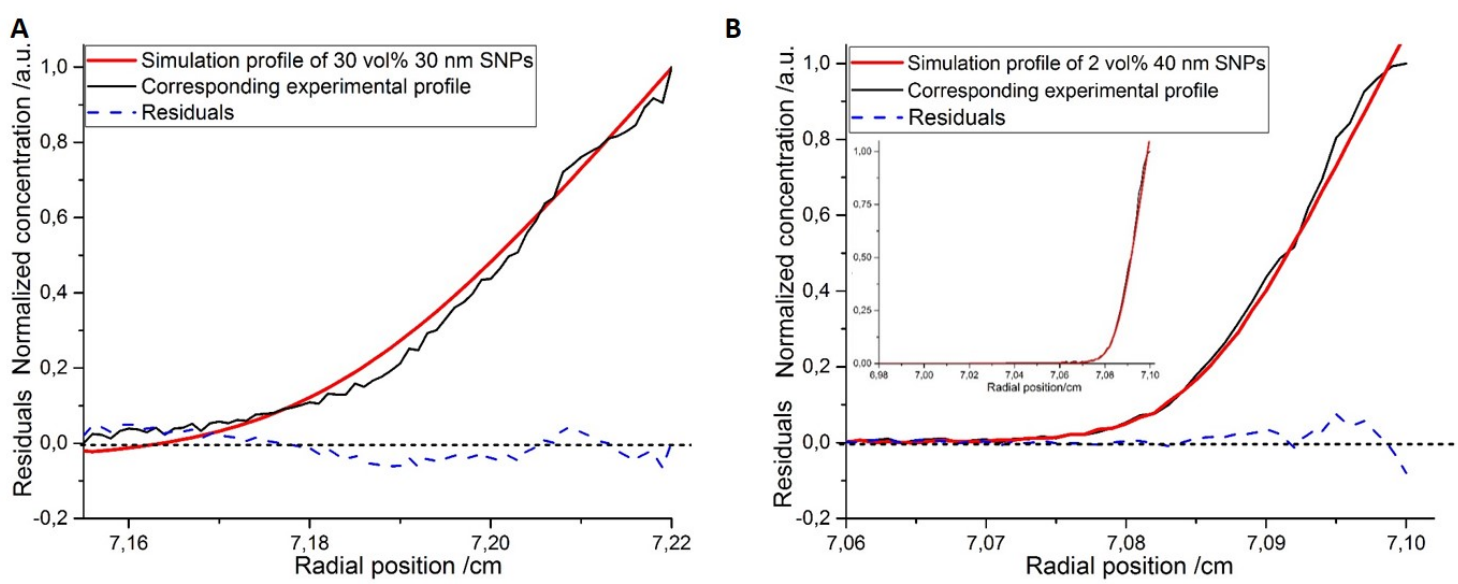

Figure 2: Normalized simulations (red solid lines) and corresponding experiments (black solid lines) for $30 \mathrm{~nm}$ RITC-SNPs of 30 vol\% (A) and $40 \mathrm{~nm}$ FITC-SNPs of 2 vol\% (B) at $2800 \mathrm{rpm}, 25^{\circ} \mathrm{C}$ (inset in (B) showed the whole sample volume). The blue dashed lines are the residuals which result from fitting to the experimental data. The experimental gradients were normalized to the range of the simulations. For (A) and $(B)$ the RMSD $=0.034$ and 0.023 respectively.

After the two monodisperse systems, we considered the binary nanoparticle mixture of $30 \mathrm{~nm}$ RITCSNPs and $40 \mathrm{~nm}$ FITC-SNPs. The concentrations profiles of these two nanoparticles were extracted separately from one absorbance spectrum (Figure S5) at every radial position in MWL-AUC (the detailed calculation procedures are described in SI6). Because these two nanoparticles were treated as hard spheres, the non-interacting model was used and thus the two concentration profiles were simulated separately using the fitted parameters for the separate nanoparticles. The 10 vol\% mixture of $30 \mathrm{~nm}$ and $40 \mathrm{~nm}$ SNPs in a volume ratio of 1:2 (Figure 3A) were simulated beforehand (another binary case with a volume ratio of 1:1 is shown in Figure S7). Then, the experiment of the same mixture was conducted. The resulting concentration gradients were normalized to compare with the simulations. We found the simulations predicted successfully the experimental results within an acceptable deviation (RMSD < 0.03). Therefore we are able to simulate and predict binary nanoparticle concentration gradients. Notably, shown in Figure 3B, the slight turbidity at very high concentration in the experiments can be 
corrected (the detailed turbidity correction procedure is described in SI7). Correspondingly, the RMSD values changed from 0.023 and 0.019 to 0.014 and 0.023 for the $30 \mathrm{~nm}$ and $40 \mathrm{~nm}$ SNPs respectively after the turbidity correction. The RMSD decreased for $30 \mathrm{~nm}$ SNPs while the RMSD increased for $40 \mathrm{~nm}$ SNPS so the remaining slight turbidity insignificantly influenced the final concentration gradients. More importantly, by the use of the confocal laser scanning microscope (CLSM), the simulations were proven valid even in preparative ultracentrifugation (PUC) (the detailed experiment and results are described in S19).

A

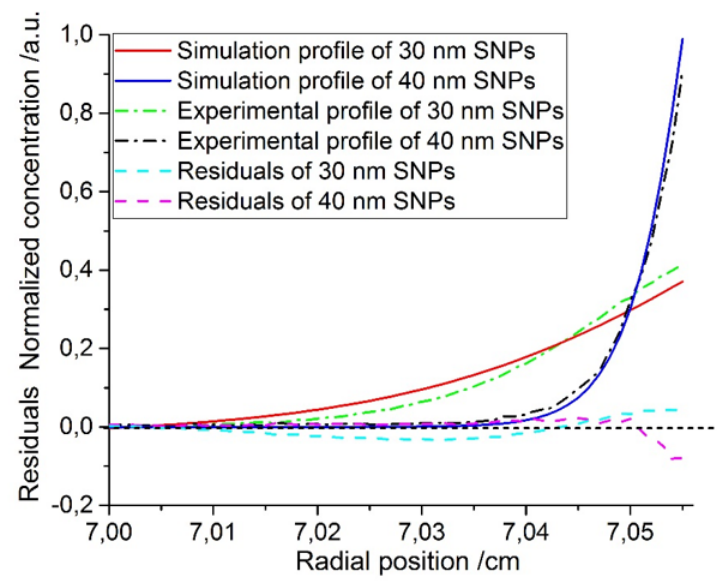

B

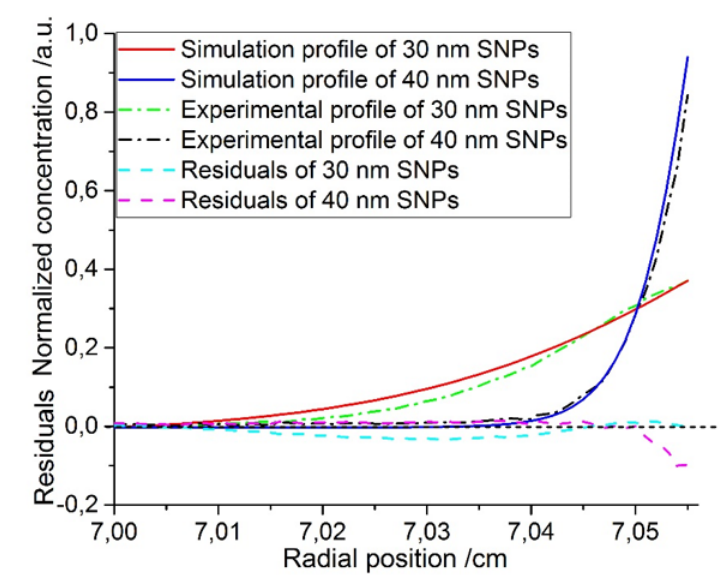

Figure 3: Normalized simulation (solid lines) and experimental (dash dotted lines) gradients for the 10 vol\% binary mixture of $30 \mathrm{~nm}$ RITC-SNPs and $40 \mathrm{~nm}$ FITC-SNPs in a volume ratio of 1:2 at $2800 \mathrm{rpm}$, $25^{\circ} \mathrm{C}(\mathrm{A})$. The slight turbidity influence at very high concentrations was corrected and a better agreement was achieved (B). The dashed lines are the residuals which result from fitting to the experimental data. The experimental concentration gradients were normalized to the range of the simulations. The RMSD values are $=0.023$ and 0.019 , respectively, for the agreement of the simulations with the experiments in $(A)$ and $=0.014$ and 0.023 , respectively, for the agreement of the simulations with the experiments in (B). 
Finally the phase behaviors for the binary mixture of $30 \mathrm{~nm}$ and $40 \mathrm{~nm}$ SNPs along the centrifugal field were studied by SEM (the detailed PUC experiment and the sample preparation for SEM are described in S110). Before the PUC experiment, the concentration gradients of the binary nanoparticle mixture were predetermined by the simulation (Figure $4 \mathrm{~A})$. From Figure $4 \mathrm{~A}$, the concentration ratios $(\approx$ volume ratio, as the two nanoparticles have the approximately same density) of the binary nanoparticle mixtures can be precisely determined to correlate with the final phase structures at each radial position (Figure 4B). In the upper part of the sediment cross-section (Figure S9) where the $30 \mathrm{~nm}$ SNPs dominated, a mixture of the $30 \mathrm{~nm}$ SNPs crystalline phase and a small amount of randomly distributed $40 \mathrm{~nm}$ SNPs were observed (Figure 5A). This phase was continuously observed from the meniscus until the radial position: $10.824 \mathrm{~cm}, 0.005 \mathrm{~cm}$ from the meniscus (Figure $\mathbf{5 B}$ and $\mathbf{C}$ ). At the positions below this transition position, where the number of $40 \mathrm{~nm}$ SNPs became comparable to that of $30 \mathrm{~nm}$ SNPs, the crystalline phase disappeared and only a glassy mixture of $30 \mathrm{~nm}+40 \mathrm{~nm}$ SNPs was observed (Figure 5D). From this continuous observation along the centrifugal field, the phase diagram was obtained (Figure 4B). The transition concentration ratio was $c_{30 \mathrm{~nm}} / c_{40} \mathrm{~nm}=4.8$ according to its corresponding radial position. When the concentration ratio was larger than 4.8, 30nm SNPs formed crystals decorated with discrete $40 \mathrm{~nm}$ SNPs while when the concentration ratio decreased from 4.8 to 1.6 at the very bottom, the two nanoparticles were mixed to form an amorphous phase.

Interestingly, a perfect agreement was found with the previous work by Hunt et al. ${ }^{25}$ They studied the size ratio $0.72 \pm 0.01$ (correspondingly a mixture of $40 \mathrm{~nm}$ and $29 \mathrm{~nm}$ particles) by light scattering, and found a crystalline phase of the smaller particle in the region $c_{S} / c_{L}>5$. When the concentration ratio decreased below 5, a fluidic-like amorphous phase was observed ${ }^{25}$. However, in the study tens of mixtures with discrete compositions were prepared while by our method, only one single sample with a continuous concentration ratio was required. 

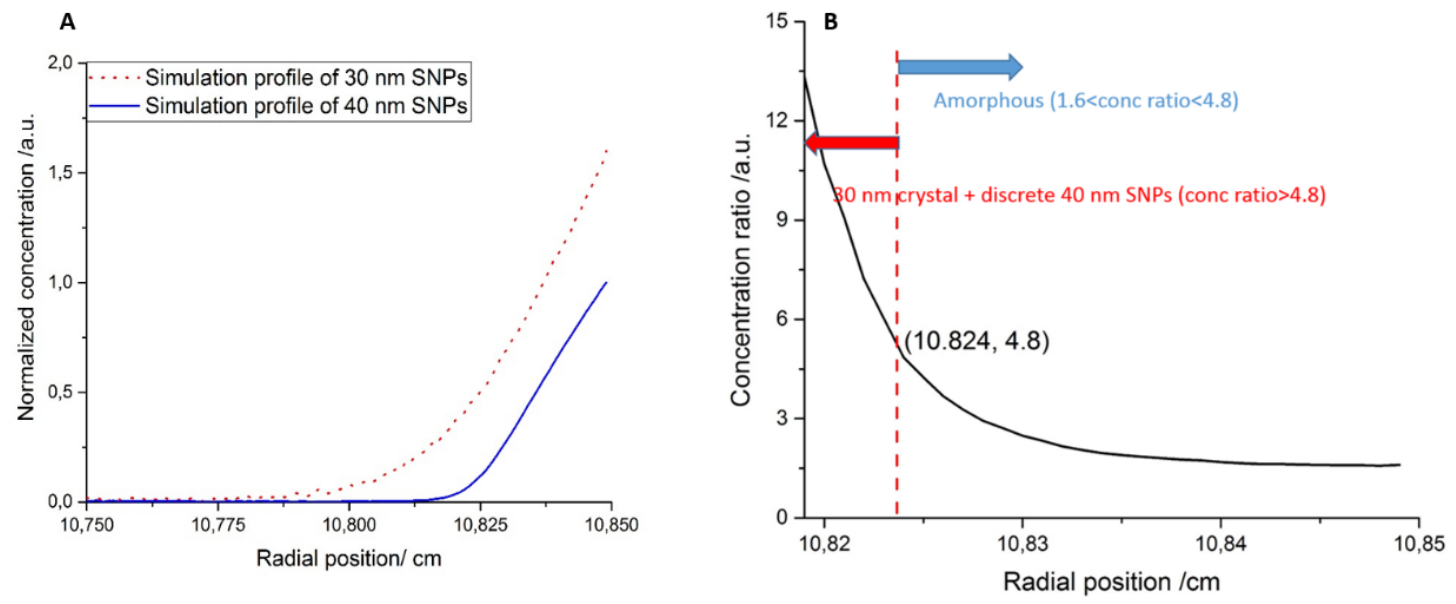

Figure 4: Normalized simulated concentration gradients for the 10 vol\% binary mixture of $30 \mathrm{~nm}$ and 40 $\mathrm{nm}$ SNPs in a concentration ratio of $4: 1$ at $2800 \mathrm{rpm}, 25^{\circ} \mathrm{C}(\mathrm{A})$. The phase diagram of the binary mixture of $30 \mathrm{~nm}$ and $40 \mathrm{~nm}$ SNPs by means of continuous observations of the sediment along the radius (B). The concentration ratio in the transition position was determined by measuring the distance from the meniscus. 

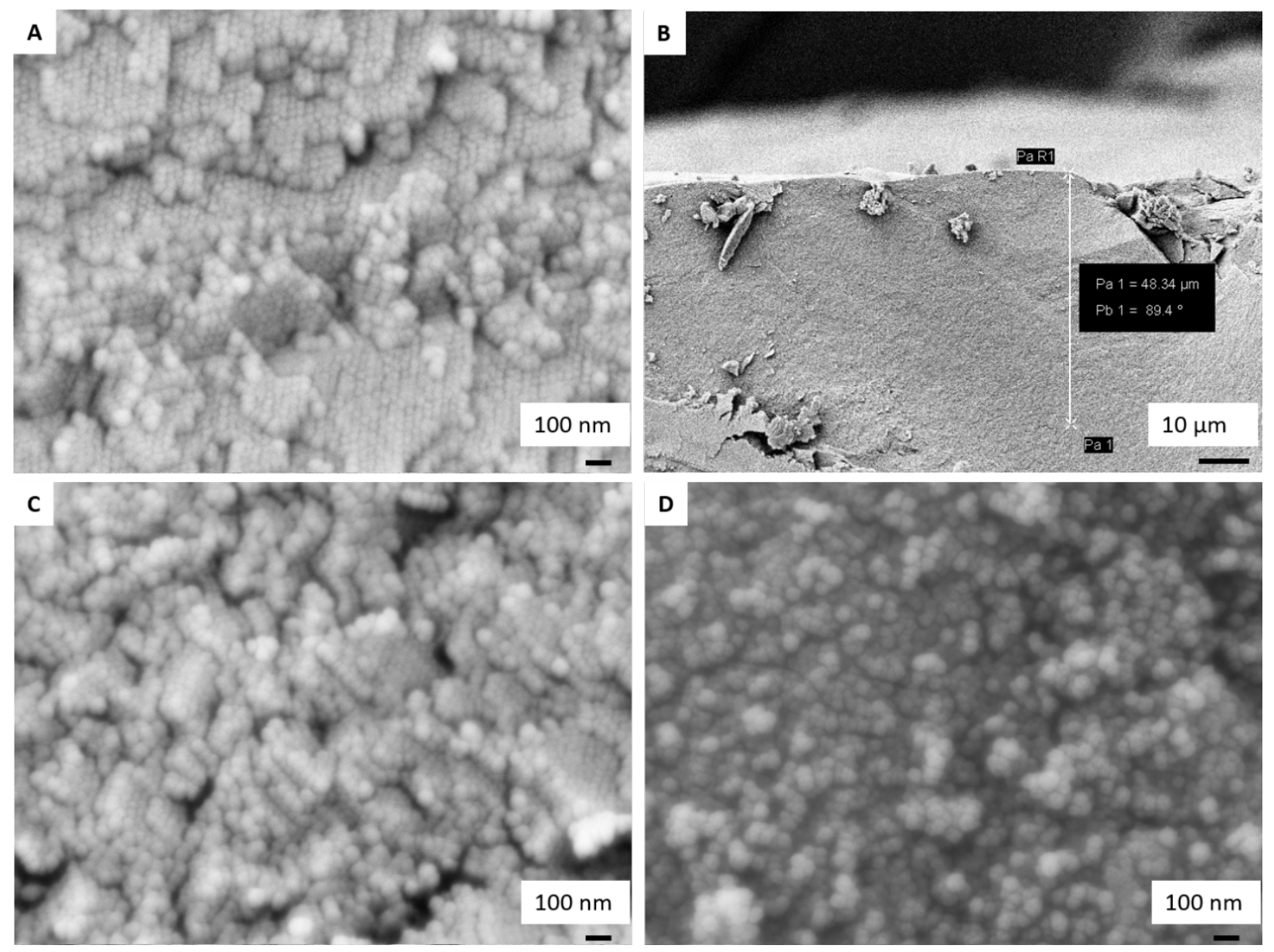

Figure 5: The represented SEM micrograph of the upper part in the sediment cross-section structures

(A). The overview of the transition position where the last piece of crystalline phase was observed $(\approx 50$ $\mu \mathrm{m}$ from the meniscus, $\mathrm{R}=10.824 \mathrm{~cm}$ ) and the radial position was determined by measuring its distance from the meniscus (B). The zoom-in SEM micrograph of the transition position (C). The represented SEM micrograph of the binary nanoparticle packing phase below the transition position (D).

In conclusion, we were able to measure binary colloidal nanoparticle concentration gradients in the AUC by the synthesis of fluorescence labeled sterically stabilized silica nanoparticles, the employment of refractive index matching solvents, the application of turbidity correction procedures and the use of a multi-wavelength analytical ultracentrifuge (MWL-AUC). The concentration gradients of moderately (circa 2 vol\%) concentrated colloids were fitted with the SEDFIT to obtain the necessary parameters including the sedimentation coefficient $s$, the diffusion coefficient $D$ as well as the non-ideality 
coefficients $k_{\mathrm{s}}$ and $k_{\mathrm{D}}$ for subsequent simulations of the concentration gradients. The simulations proved to be valid even for very high particle concentrations up to 30 vol\%. In the future, an even higher concentration may be studied to check the simulation validity limit. With this knowledge, we are now able to simulate and tailor-make binary colloidal nanoparticle concentration gradients in a centrifugal field. Moreover the simulated concentration gradients can be used to correlate with different binary nanoparticle phases along the centrifugal field so that a continuous and extended binary nanoparticle phase diagram can be obtained simply by studying one single sample by SEM. In the future we will study size ratios with a richer phase behavior, especially in the size ratio regime $0.5-0.6^{25,50}$. These studies will help to explore a large variety of binary nanoparticle phases including different crystalline and amorphous structures, which can be formed simultaneously using a preparative ultracentrifuge, in one single sample. The simulations of the concentration gradients can be applied as long as the colloids can be treated as hard spheres. Moreover nanoparticles of different shapes such as rod-like, platelet-like can be studied using the same methodology. In principle, different shapes affect the frictional ratio $\left(f / f_{0}\right)$, which in turn influences the sedimentation and diffusion coefficients ( $s$ and $D$ ). These two coefficients can be determined by AUC-SV experiments shown in SI4 and are input parameters for the simulations. Overall, our study of binary nanoparticle concentration gradients in a centrifugal field at high concentration shows a promising future in the research on the phase diagram in a continuous and handy manner for nanoparticle mixtures of multiple different sizes.

\section{ASSOCIATED CONTENT}

\section{Supporting Information}

The following files are available free of charge. 
The synthesis process of $30 \mathrm{~nm}$ and $40 \mathrm{~nm}$ fluorescence labeled PEG stabilized silica nanoparticles (SI1); Images of the nanoparticle dispersions before and after refractive index matching and the calculation procedures to prove that the glycerol-water mixture formed a negligible density gradient in the AUC (SI2); The detailed AUC sedimentation-diffusion equilibrium experimental set-up (SI3); The detailed fitting process (SI4); The calculation procedures of the density $(\rho)$ and molecular mass $(M)$ for $30 \mathrm{~nm}$ and $40 \mathrm{~nm}$ SNPs (SI5); The calculation procedures of separate nanoparticle concentrations from the absorbance spectra of the binary nanoparticle mixture (SI6); The turbidity correction procedure (SI7); Another simulation result for the binary case with a volume ratio of 1:1 (SI8). The confocal laser scanning microscope (CLSM) experiment to prove the validity of the simulation in PUC (SI9). The PUC experiment and the sample preparation for SEM (SI10).

\section{AUTHOR INFORMATION}

\section{Corresponding Author}

*E-mail: helmut.coelfen@uni-konstanz.de

\section{Author Contributions}

N.S. led the project. H.C. conceived the idea and supervised the experiments and simulations. K.S. designed and supervised the AUC experiments. X.X. synthesized the nanoparticles, X.X. and T.F. conducted the AUC experiments, X.X. analyzed the AUC experimental data and conducted the Sedfit simulation. X.X. conducted the PUC experiments. X.X. wrote the manuscript. All authors discussed the results and the manuscript.

\section{Notes}

The authors declare no competing financial interest. 


\section{ACKNOWLEDGMENT}

The authors would like to acknowledge the financial support from the EU H2020-MSCA-ITN2015 project 'MULTIMAT' (project number: 676045). We also thank Martin Stöckl and Elisa

May from the Bioimaging Center, Universität Konstanz for their generous assistance with the confocal laser scanning microscope experiments.

References:

(1) Howes, P. D.; Chandrawati, R.; Stevens, M. M. Science 2014, 346, 1247390.

(2) Parak, W. J.; Gerion, D.; Pellegrino, T.; Zanchet, D.; Micheel, C.; Williams, S. C.; Boudreau, R.; Le Gros, M. A.; Larabell, C. A.; Alivisatos, A. P. Nanotechnology 2003, 14, R15.

(3) Huh, S.; Chen, H.-T.; Wiench, J. W.; Pruski, M.; Lin, V. S. Y. Angewandte Chemie International Edition 2005, 44, 1826-1830.

(4) Mu, Y.; Liang, H.; Hu, J.; Jiang, L.; Wan, L. The Journal of Physical Chemistry B 2005, 109, 2221222216.

(5) Chan, K.-Y.; Ding, J.; Ren, J.; Cheng, S.; Tsang, K. Y. Journal of Materials Chemistry 2004, 14, 505516.

(6) Vogel, N.; Retsch, M.; Fustin, C.-A.; del Campo, A.; Jonas, U. Chemical reviews 2015, 115, 62656311.

(7) Li, F.; Josephson, D. P.; Stein, A. Angewandte Chemie International Edition 2011, 50, 360-388.

(8) Joannopoulos, J. D.; Villeneuve, P. R.; Fan, S. Nature 1997, 386, 143.

(9) Hynninen, A.-P.; Thijssen, J. H.; Vermolen, E. C.; Dijkstra, M.; Van Blaaderen, A. Nature materials 2007, 6, 202.

(10) Shevchenko, E. V.; Talapin, D. V.; Kotov, N. A.; O'Brien, S.; Murray, C. B. Nature 2006, 439, 55-9.

(11) Leunissen, M. E.; Christova, C. G.; Hynninen, A. P.; Royall, C. P.; Campbell, A. I.; Imhof, A.;

Dijkstra, M.; van Roij, R.; van Blaaderen, A. Nature 2005, 437, 235-40.

(12) Chen, M.; Cölfen, H.; Polarz, S. ACS nano 2015, 9, 6944-50.

(13) Shevchenko, E. V.; Talapin, D. V.; Murray, C. B.; O'Brien, S. Journal of the American Chemical Society 2006, 128, 3620-3637.

(14) Filion, L.; Dijkstra, M. Physical Review E 2009, 79, 046714.

(15) Zunger, A. Physical Review B 1980, 22, 5839.

(16) Bartlett, P.; Campbell, A. I. Physical review letters 2005, 95, 128302.

(17) Bartlett, P.; Pusey, P. N. Physica A: Statistical Mechanics and its Applications 1993, 194, 415-423.

(18) Murray, M.; Sanders, J. Philosophical Magazine A 1980, 42, 721-740.

(19) Dong, A.; Chen, J.; Vora, P. M.; Kikkawa, J. M.; Murray, C. B. Nature 2010, 466, 474.

(20) Meder, F.; Thomas, S. S.; Bollhorst, T.; Dawson, K. A. Nano letters 2018, 18, 2511-2518.

(21) Mann, S. Nature materials 2009, 8, 781.

(22) Lu, P. J.; Weitz, D. A. Annual Review of Condensed Matter Physics 2013, 4, 217-233.

(23) Schuck, P.; Zhao, H.; Brautigam, C. A.; Ghirlando, R., Basic principles of analytical ultracentrifugation. CRC Press: 2016.

(24) Fujita, H., Foundations of ultracentrifugal analysis. Wiley: 1975. 
(25) Hunt, N.; Jardine, R.; Bartlett, P. Physical Review E 2000, 62, 900-913.

(26) Royall, C. P.; Vermolen, E. C.; van Blaaderen, A.; Tanaka, H. Journal of Physics: Condensed Matter 2008, 20, 404225.

(27) Pearson, J.; Walter, J.; Peukert, W.; Cölfen, H. Analytical chemistry 2018, 90, 1280-1291.

(28) Lamm, O. Zeitschrift für Physikalische Chemie 1929, 143, 177-190.

(29) Schuck, P. Biophysical journal 2000, 78, 1606-19.

(30) Van Blaaderen, A.; Vrij, A. Langmuir 1992, 8, 2921-2931.

(31) Watanabe, R.; Yokoi, T.; Kobayashi, E.; Otsuka, Y.; Shimojima, A.; Okubo, T.; Tatsumi, T. Journal of colloid and interface science 2011, 360, 1-7.

(32) Ma, K.; Mendoza, C.; Hanson, M.; Werner-Zwanziger, U.; Zwanziger, J.; Wiesner, U. Chem Mater 2015, 27, 4119-4133.

(33) Verhaegh, N. A. M.; Vanblaaderen, A. Langmuir 1994, 10, 1427-1438.

(34) Yokoi, T.; Sakamoto, Y.; Terasaki, O.; Kubota, Y.; Okubo, T.; Tatsumi, T. Journal of the American Chemical Society 2006, 128, 13664-13665.

(35) Ma, K.; Zhang, D.; Cong, Y.; Wiesner, U. Chem Mater 2016, 28, 1537-1545.

(36) Ow, H.; Larson, D. R.; Srivastava, M.; Baird, B. A.; Webb, W. W.; Wiesner, U. Nano letters 2005, 5, 113-117.

(37) Herz, E.; Ow, H.; Bonner, D.; Burns, A.; Wiesner, U. Journal of Materials Chemistry 2009, 19, 6341-6347.

(38) Yethiraj, A.; van Blaaderen, A. Nature 2003, 421, 513.

(39) Maechtle, W.; Börger, L., Analytical ultracentrifugation of polymers and nanoparticles. Springer: Berlin ; New York, 2006.

(40) Planken, K. L.; Cölfen, H. Nanoscale 2010, 2, 1849-1869.

(41) Laue, T.; Stafford III, W. Annual review of biophysics and biomolecular structure 1999, 28, 75-

100.

(42) Cole, J. L.; Lary, J. W.; Moody, T. P.; Laue, T. M. Methods in cell biology 2008, 84, 143-179.

(43) Karabudak, E.; Cölfen, H., The Multiwavelength UV/Vis Detector: New Possibilities with an Added Spectral Dimension. In Analytical Ultracentrifugation: Instrumentation, Software, and Applications, Springer Japan: Tokyo, 2016.

(44) Walter, J.; Löhr, K.; Karabudak, E.; Reis, W.; Mikhael, J.; Peukert, W.; Wohlleben, W.; Cölfen, H. ACS nano 2014, 8, 8871-8886.

(45) Vistica, J.; Dam, J.; Balbo, A.; Yikilmaz, E.; Mariuzza, R. A.; Rouault, T. A.; Schuck, P. Analytical Biochemistry 2004, 326, 234-256.

(46) Behlke, J.; Ristau, O. Biophysical chemistry 1999, 76, 13-23.

(47) Laue, T. M., [19] Sedimentation equilibrium as thermodynamic tool. In Methods in enzymology, Elsevier: 1995.

(48) Carney, R. P.; Kim, J. Y.; Qian, H.; Jin, R.; Mehenni, H.; Stellacci, F.; Bakr, O. M. Nature communications 2011, 2, 335.

(49) Page, M. G.; Zemb, T.; Dubois, M.; Cölfen, H. ChemPhysChem 2008, 9, 882-890.

(50) Bartlett, P.; Ottewill, R. H.; Pusey, P. N. Physical Review Letters 1992, 68, 3801-3804. 